\title{
Spinal Epidural Abscesses
}

\section{Sonia G. Teufack, MD}

Department of Neurological Surgery, Thomas Jefferson University, Philadelphia, PA

Despite advances in the diagnosis and treatment of neurosurgical diseases, spinal epidural abscesses (SEA) remain challenging. The diagnosis is complex, treatments are controversial, and the potential for adverse outcomes is significant.

SEA accounts for 2 of every 10,000 of hospital admissions, an incidence which has doubled in the past twenty years. Reasons which may account for this include an aging population, increased IV drug use, and increase in number of vascular and spinal procedures.

SEA can arise from an underlying medical condition, such as diabetes mellitus, alcoholism, chronic obstructive pulmonary disease (COPD), or HIV infection. Loci for SEA can be formed by other spinal abnormalities or by prior invasive spinal procedures, including epidurals, nerve blocks, or steroid injections. Sources for systemic infection may include vascular access catheters, IV drug use, or chronic UTI. 50\% of spinal epidural infections have hematogenous origin, $30 \%$ arise from the skin or connective tissue, and $20 \%$ are unclassified. Common pathogens include S. aureus and $P$. aeruginosa. From $15 \%$ to $40 \%$ of SEA may be due to MRSA. In cases of spine trauma or spinal surgery, S. epidermidis may also be a pathogen.

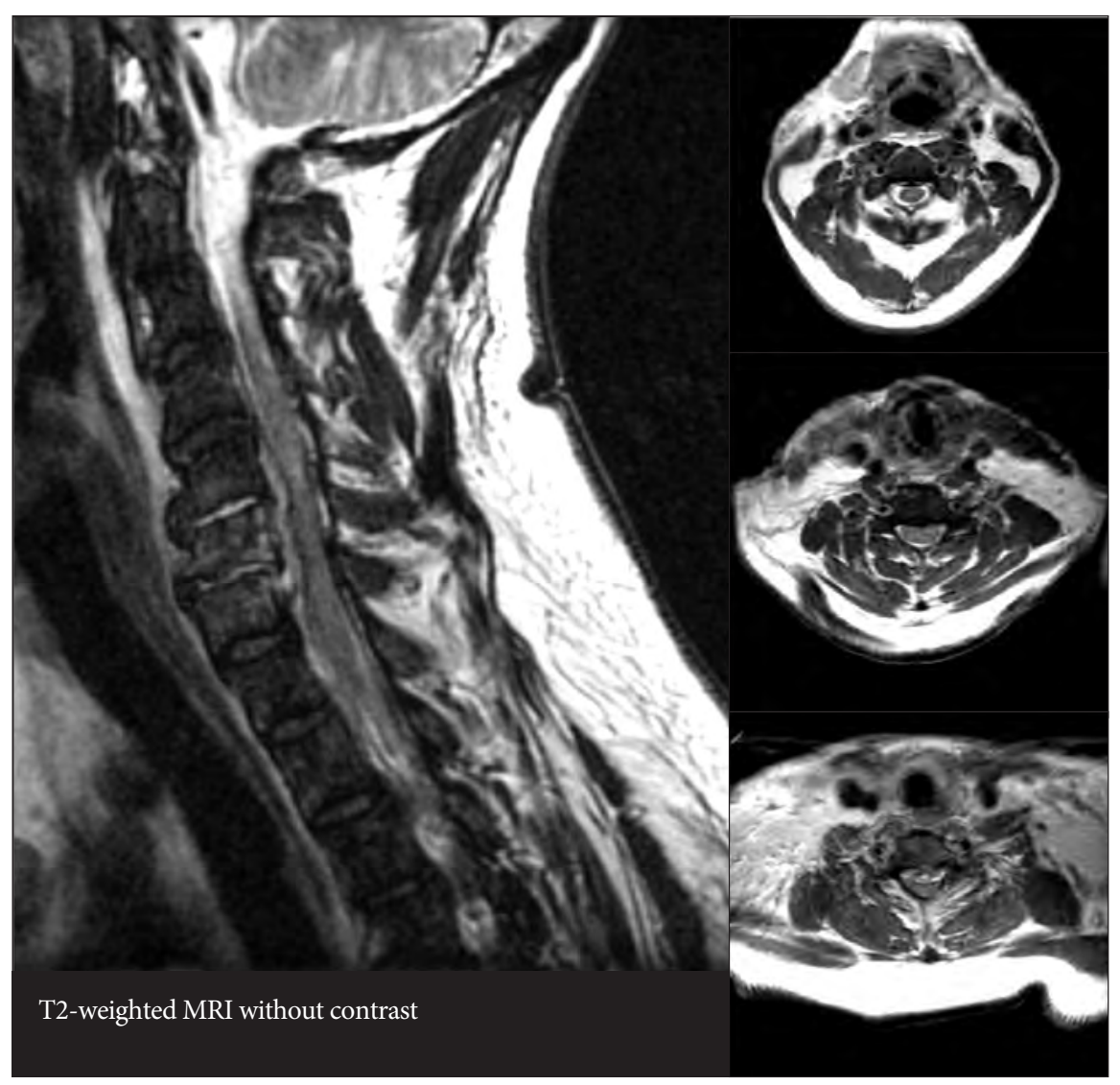

$79 \%$ of SEA appear dorsal to the spinal cord. A possible explanation for this finding is that the largest extent of the epidural space lies posterior to the nerve roots. The $21 \%$ that appear anterior are often associated with vertebral discitis and/ or osteomyelitis.

Achieving correct diagnosis in cases of SEA may be challenging. $50 \%$ of epidural spinal abscesses are initially misdiagnosed. The most common presenting symptom is back pain, present in up to $85 \%$ of patients. Fever is present in up to $50 \%$. Less common symptoms are paresthesias, sensory deficits, radicular pain, or motor deficits. SEA can occur spontaneously in patients with increased co-morbidities. In post-operative patients, SEA may take days to weeks to appear. A dorsal SEA results from the spontaneous seeding of the dorsal epidural fat. As the abscess enlarges, neural compression may occur. Conversely, a ventral SEA may result from either spontaneous seeding of the ventral epidural fat or seeding of the disc space with secondary extension into the ventral epidural space. A ventral SEA is more likely to present with systemic symptoms (i.e., fevers, septicemia) prior to presentation of neurological deficits.

The best way to diagnose SEA is to approach high-risk patients with suspicion. The neurological exam may aid in localizing the level of spinal involvement in cases with neurological deficit. Laboratory evaluations should include ESR, CRP and pan cultures. Elevated ESR has high specificity. $S$. aureus bacteremia, as an isolated finding, does not decisively establish the source of infection. CSF sampling risks of introduction of infected material into the subarachnoid and/or subdural space. CSF analysis should show increased protein and pleocytosis, gram stain is usually negative, and $17-25 \%$ of CSF cultures are positive.

$\mathrm{X}$-Rays have poor sensitivity and specificity. Basic radiographic exams frequently appear normal unless there is associated osteomyelitis and/or discitis with significant bony erosion. CT scans without contrast similarly have a high rate of false negatives. With contrast, CT may show loss of epidural fat and contrast fixation at the level of the abscess. Myelography 


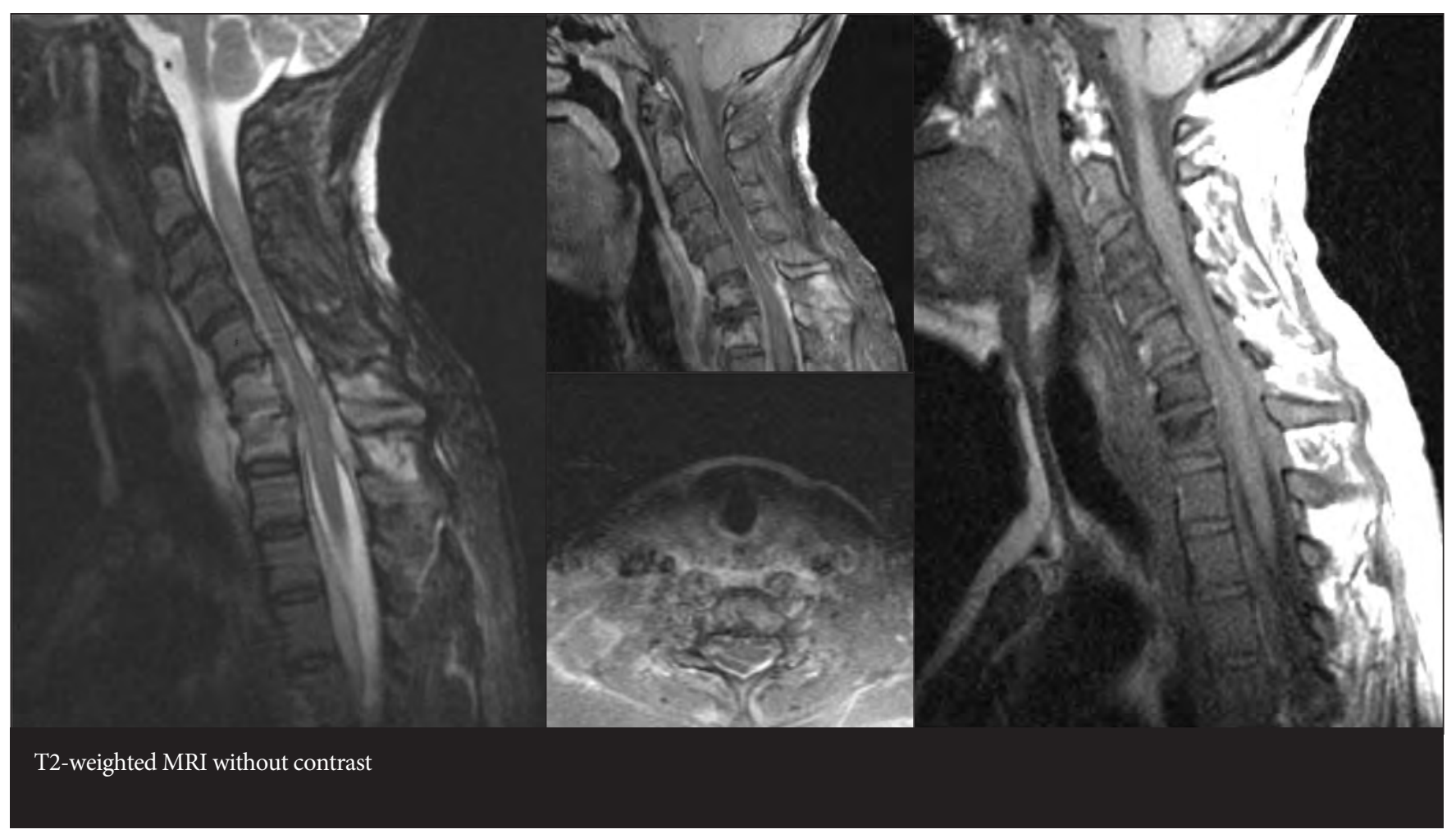

was previously the gold standard and is still used for patients that cannot undergo MRI. Myelography can detect high to complete block of CSF flow and can establish whether the abscess is anterior or posterior to the neural elements.

T2-weighted MRI without contrast has diagnostic efficacy in SEA, but also a high rate of false negatives. False negatives may occur due to poor differentiation between CSF and SEA signals, especially with very extensive abscesses, and poor differentiation between infected CSF and SEA in patients with meningitis. Contrasted MRI has greatest utility in delineating SEA extent. There is still the risk of a false positives, through misreading of epidural venous plexus, and false negatives, possible in a patients with epidural lipomatosis.

There are no conclusive guidelines present in the literature for treatment of SEA. There is no RCT comparing medical management to surgical intervention, and there is considerable variation in practices. With one exception ${ }^{1}$, most retrospective studies ${ }^{3,5-7}$ indicate that the majority of SEA patients may be treated conservatively. Among these studies, the consensus is conservative treatment (IV antibiotic with or without CT guided needle aspiration) if the patient is neurologically intact or if the neurological deficit has been stable for over 72 hours, with surgery reserved for cases of neurological deterioration.

Rigamonti et $\mathrm{al}^{5}$ found that thoracic SEA was likelier to result in poor outcomes than SEA located elsewhere (36\% vs $10 \%)$, and that patients surgically treated within 24 hours of diagnosis were less likely to have poor outcomes than patients treated later (10\% vs. $47 \%)$. Curry also reported that SEA patients receiving surgery within 12 hours did better than patients treated conservatively. Karikari et $\mathrm{al}^{3}$ did not see a difference in timing of surgical treatment, but did find that ventral vs. dorsal location of SEA was associated with differing rates of para/ quadriplegia (7.3\% vs. $30.6 \%$ ); with Rigamonti's report, this indicates anatomy of the SEA should be considered in a treatment plan. Siddiq et $\mathrm{al}^{7}$ found no clear difference between surgery and conservative treatment, but reported that greater neurologic abnormality at presentation was associated with greater likelihood of poor outcome.

In medical management, the priorities are to establish the pathogen and source of infection through biopsy with cultures and to provide appropriate, organism-specific anti- microbial coverage. There should be frequent neurological exams and aggressive physical therapy, occupational therapy and rehab.

The main goals of surgical management of SEA are to prevent further neurological deterioration and to achieve early spinal cord decompression and structural stabilization. Laminectomy is the treatment of choice for dorsal SEA; decompression and stabilization through anterior column debridement and reconstruction are completed for ventral SEA with vertebral osteomyelitis and/or discitis.

The natural history of untreated SEA is progression of symptoms to paralysis and potentially death. Before the advent of antibiotics the mortality rate in SEA was $55-70 \%$. With the advent of surgery in the 1930s, the mortality decreased to $30-57 \%$. Current mortality rates are $2-20 \%$. Neurological recovery depends on the severity and duration of symptoms before treatment. $4-22 \%$ of SEA develop irreversible paralysis while $0-4 \%$ of SEA develop recurrence.

Continued improvement of outcomes in SEA requires education of primary care providers in realization and appropriate evaluation of high risk patients, prompt invasive testing to achieve definitive diagnosis, and a multidisciplinary approach to treatment. 


\section{References}

1. Curry WT Jr, Hoh BL, Amin-Hanjani S, Eskandar EN. Spinal epidural abscess: clinical presentation, management, and outcome. Surg Neurol. 2005 Apr;63(4):364-71

2. Darouiche RO. Spinal epidural abscess. N Engl J Med. 2006 Nov 9;355(19):2012-20.

3. Karikari IO, Powers CJ, Reynolds RM, Mehta AI, Isaacs RE. Management of a spontaneous spinal epidural abscess: a single-center 10-year experience. Neurosurgery. 2009 Nov;65(5):919-23; discussion 923-4.

4. Pradilla G, Ardila GP, Hsu W, Rigamonti D. Epidural abscesses of the CNS. Lancet Neurol. 2009 Mar;8(3):292-300.

5. Rigamonti D, Liem L, Sampath P, Knoller N, Namaguchi Y, Schreibman DL, Sloan MA, Wolf A, Zeidman S. Spinal epidural abscess: contemporary trends in etiology, evaluation, and management. Surg Neurol. 1999 Aug;52(2):189-96

6. Savage K, Holtom PD, Zalavras CG. Spinal epidural abscess: early clinical outcome in patients treated medically. Clin Orthop Relat Res. 2005 Oct;439:56-60.

7. Siddiq F, Chowfin A, Tight R, Sahmoun AE, Smego RA Jr. Medical vs surgical management of spinal epidural abscess. Arch Intern Med. 2004 Dec 13-27;164(22):2409-12

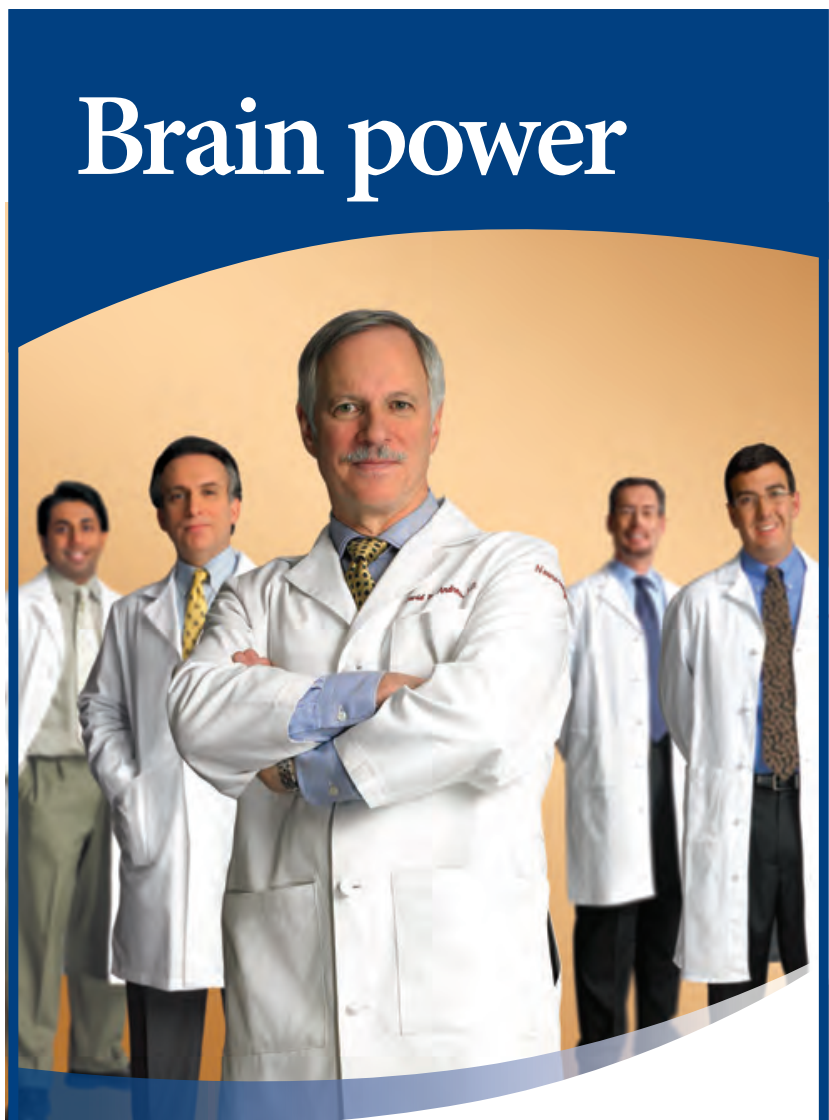

Jefferson neurosurgeons have helped to write the textbooks and set training standards referenced by other doctors around the world. No wonder our surgeons treat the largest combined volume of tumors, aneurysms and AVMs in the Philadelphia region.

The region's only hospital with an experienced, specialized staff dedicated to neuroscience.

Offices in Center City Philadelphia, Langhorne and Voorhees. Most insurance plans accepted, including Medicare.

\section{Jefferson.}

\section{Hospital for Neuroscience}

- Thomas Jefferson University Hospital

- Jefferson Hospital for Neuroscience

- Methodist Hospital 